\title{
Un centre de ressources au service d'une approche méthodologique de la compréhension de l'anglais oral pour un public de DEUG indifférencié
}

Jean Sabiron

\section{(2) OpenEdition}

\section{Journals}

Édition électronique

URL : http://journals.openedition.org/asp/3759

DOI : 10.4000/asp.3759

ISSN : 2108-6354

Éditeur

Groupe d'étude et de recherche en anglais de spécialité

\section{Édition imprimée}

Date de publication : 1 décembre 1995

Pagination : 145-161

ISSN : 1246-8185

\section{Référence électronique}

Jean Sabiron, « Un centre de ressources au service d'une approche méthodologique de la compréhension de l'anglais oral pour un public de DEUG indifférencié », ASp [En ligne], 7-10 | 1995, mis en ligne le 24 septembre 2013, consulté le 01 mai 2019. URL : http://journals.openedition.org/ asp/3759 ; DOI : 10.4000/asp.3759

Ce document a été généré automatiquement le 1 mai 2019.

Tous droits réservés 


\title{
Un centre de ressources au service d'une approche méthodologique de la compréhension de l'anglais oral pour un public de DEUG indifférencié
}

\author{
Jean Sabiron
}

1 Le but de la présente communication est de rendre compte d'un cheminement méthodologique, au cours des quatre dernières années au sein de l'Université de Poitiers, et de définir le concept d'un Centre de ressources au service de grands nombres d'étudiants de DEUG, spécialistes de la langue anglaise et spécialistes d'autres disciplines.

2 Notre recherche en didactique qui a donné lieu à soutenance d'une thèse de type «Recherche-Action » a pour objet de rendre un système de formation plus efficace, en proposant des solutions nouvelles, ancrées dans une approche théorique, et validées dans une approche scientifique expérimentale.

3 Le domaine du praticien et du chercheur, en l'occurrence, est celui de la compréhension orale, et plus précisément le perfectionnement de cette compétence, dans le cadre institutionnel existant, c'est-à-dire non exolingue.

4 Nous proposons d'aborder successivement la mise en œuvre d'un outil didactique visant à l'acquisition quantitative d'un contenu linguistique autour du matériau et de la démarche VIFAX conçue par M. Perrin, de l'Université Bordeaux 2, et le rôle du Centre dans cette phase.

5 En second lieu, après avoir rappelé l'ancrage théorique cognitif qui est à la base de cette évolution et défini ce qu'est la compréhension orale selon cette approche, nous présentons le concept d'ACADEME : cet outil méthodologique intégré répond à l'objectif immédiat de culture langagière, et introduit la culture méthodologique.

6 En troisième partie, nous définissons la nature des tâches du Centre de ressources dans cette $2^{\mathrm{e}}$ phase, les conséquences méthodologiques des orientations que ce Centre peut 
susciter dans l'institution universitaire: à savoir une formation et une pratique qualitatives nécessaires à l'autonomie des étudiants, dès la phase de formation à l'université, et la mise en œuvre d'une alternance progressive d'activités présentielles et d'activités en autonomie.

\section{Perfectionner la compréhension de l'oral}

7 La mise en œuvre de VIFAX constitue une proposition langagière répondant à l'urgence de perfectionnement de la compréhension orale, «l'aptitude oubliée » (Holec 1990 : 30).

\subsection{Historique}

$8 \quad$ Face aux effectifs pléthoriques de DEUG Sciences et surtout de l'UFR Lettres et Langues, il était devenu urgent de réfléchir à une exposition cohérente et systématique à la langue anglaise, commune à tous les groupes d'un même niveau de formation. Depuis 2 ans, CRESSIDA (notre Centre de ressources et de services intégrés d'anglais) a pris l'initiative de proposer à ces 2 publics (220 étudiants en DEUG B2, et 800 en $1^{\text {re }}$ année d'anglais LEA et LCE) un corpus sonore constitué exclusivement de documents audiovisuels récents, variés, et représentatifs de la langue et de la culture étrangères, sélectionnés par les concepteurs de VIFAX à Bordeaux.

Dès 1993, nous avions formulé l'hypothèse que le contenu d'une seule année de ces programmes (240 documents, à raison de 8 par semaine, pendant 30 semaines) pouvait suffire à alimenter en matériau sonore de qualité, les 25 semaines de cours d'étudiants spécialistes ou non (à raison d'un document nouveau par semaine). CRESSIDA a donc analysé les 240 documents des 10 mois précédents, en provenance alternée des chaînes de télévision britannique et américaine Skynews et CNN. Ceux-ci ont fait l'objet d'une sélection en 2 étapes et ont permis de constituer un corpus de 25 documents qui a donné lieu à une étude systématique dans le cadre de l'UV de "Compréhension orale » des étudiants d'anglais 1re année, selon la démarche suivante: en cours, visionnement du document vidéo, présentation d'une fiche de travail d'audition autonome : identification, repérage d'éléments lexicaux ou phonologiques (la composante " phonétique » de cette U.V. étant articulée partiellement autour de ce même matériau.), et questions de synthèse à traiter à domicile pour la semaine suivante (chaque étudiant dispose en effet de sa propre cassette audio de la bande-son du document visionné) ; au début du cours suivant : mise en commun des informations recueillies, correction, et distribution de la transcription du document.

\subsection{Pourquoi le Centre de ressources a choisi VIFAX}

Tout d'abord, les collègues de VIFAX opèrent un premier filtre de 120 fois 30 minutes par an; ces anglophones vivent en France, et sont capables de relativiser leur propre culture et de saisir l'événement ou le repère qui l'illustre.

11 Outre ce travail de sélection initiale, le concept VIFAX propose la didactisation spontanée et de qualité, s'adressant à des adultes, de 2 documents quotidiens en provenance de 2 chaînes anglaise et américaine ( 3 feuilles par document retenu : feuille d'exercice, corrigé de cette feuille, et scriptage) utilisable telle quelle par des étudiants en groupes ou isolés ; la transmission pratique de ces documents de travail par fax et surtout par modem, ${ }^{1} \mathrm{ce}$ 
qui facilite énormément le traitement de ces documents. Comme ces chaînes sont non cryptées, chaque abonné les capte sur ses propres installations de réception, il n'y a donc ni duplication ni expédition du matériau audio-visuel de base. Le type de message diffusé par ces chaînes et retenu par VIFAX et enregistré simultanément par tous les abonnés est d'une longueur acceptable (2 à 3 minutes), c'est un document authentique d'actualité, en provenance de Grande-Bretagne ou des États-Unis; il présente une constance dans sa macro-structure (introduction claire et développement redondant en plusieurs étapes), une alternance entre un discours lu par un journaliste professionnel, et le discours direct des personnes interviewées. La clarté entre les formes de surface et les significations sous-jacentes permet que la première interprétation soit la seule cohérente avec le principe de pertinence : la facilité de traitement des indices dans la visée d'apprentissage épargne ainsi les efforts inutiles de différentes interprétations ou de dispersion. Enfin, la complémentarité image - son incite à l'heuristique des liens de causalité.

12 La production d'une seule année (240 documents) permet la constitution dans la durée d'un fonds documentaire audiovisuel de plus de 9 heures, référencé, vaste et varié, pouvant répondre à des besoins ultérieurs d'enseignement (matériau d'apprentissage et d'évaluation sur les mêmes thèmes), et des besoins de corpus de recherche.

13 Enfin, les anglophones qui didactisent quotidiennement ces documents audiovisuels travaillent au sein d'une équipe de recherche dynamique, qui s'intéresse notamment à la didactique des langues pour les spécialistes d'autres disciplines (LANSAD), population universitaire environ trois fois plus importante que celle des UFR de Langues.

\subsection{Les rôles du Centre de ressources}

Un traitement matériel et technique de la masse documentaire: acquisition ou réception, identification, vérification, cotation, évaluation, classement, et stockage, « en vue de fournir à un groupe d'utilisateurs particuliers une information convenablement “dirigée" sur une base régulière " selon la formulation de l'UNESCO en 1971 (Fondin 1992).

15 Un traitement intellectuel (linguistique et didactique) des documents: en 1992-93, CRESSIDA a sélectionné pour un public de DEUG indifférencié 58 documents VIFAX sur 152 reçus; en 1993-94, 84 documents sur les 240 annuels soit $35 \%$, selon les critères suivants.

\section{Critère du matériau ou du support}

Qualité du son et de l'image (au niveau création, diffusion, enregistrement) puisqu'ils vont être dupliqués à plusieurs niveaux, (le document vidéo et sonore proposé à l'étude est le document primaire ou brut non traité).

\section{Critère du contenu}

17 - Critères langagiers : qualité linguistique et phonologique ;

18 - Critère de difficulté ou plutôt d'accessibilité : un document peut être à la fois authentique et accessible ;

19 - Critère temporel : intérêt pérenne du document, information durable ou permanente qui fait partie du stock des connaissances humaines, qui permettent une certaine distance historique ; de plus pour des raisons d'organisation on ne peut utiliser que du différé d'un an ; 
20 - Critères culturels : représentativité de la culture, donc une rupture (notion d'acculturation de McLaughlin); cette représentativité est soit prototypique ou pertinente, soit anecdotique: un événement discret, une information éphémère conjoncturelle seront retenus s'ils illustrent une problématique de la «culture de l'autre ";

21 - Critère de comparaison, de mise en relations des faits actuels avec ceux d'autres pays, ou d'autres périodes de ce même pays ;

22 - Une volonté de rupture avec les thèmes récurrents en second cycle du secondaire, dont la vision optimiste du monde n'est pas la caractéristique première :

23 - Des exemples de progrès (scientifique ou technologique, ou tout simplement sur la cohabitation des peuples) ;

24 - Une visée instrumentale prévisible: en direction de scientifiques, de juristes, d'économistes, de littéraires, d'élèves ingénieurs, etc. ;

25 - Une visée récréative : des documents publicitaires (entre autres) y sont propices.

26 À quels publics de l'université s'adressent les 84 documents ?

Tableau1. Documents et publics

\begin{tabular}{|l|l|}
\hline Total documents & 84 \\
\hline s'adressent à toutes catégories d'étudiants & 46 \\
\hline principalement à des étudiants scientifiques et/ou de médecine & 17 \\
\hline principalement à des juristes & 8 \\
\hline principalement à des économistes & 13 \\
\hline
\end{tabular}

27 Le troisième rôle de CRESSIDA concerne la diffusion des documents :

28 - Documents bruts non traités, en Lettres, droit, IUT GEA, École d'ingénieurs. Ces publics reçoivent une bande vidéo, une bande-son à part, le scriptage (qui sera distribué aux étudiants après l'étude du document sonore), et les 2 feuilles d'exercices VIFAX permettant d'inspirer les concepteurs de feuilles de compréhension propres à l'établissement.

29 - En revanche, en Sciences, les documents sont traités avant diffusion : adaptation des feuilles de travail, et des scripts (en version grammaticale, ou lexique scientifique; versions lacunaires, etc.), pour des groupes ou des étudiants isolés.

Enfin, le Centre de ressources élabore une évaluation du matériau et de la méthodologie auprès des utilisateurs (étudiants et enseignants) :

31 Cette expérience a donné lieu à une enquête détaillée dont il est opportun d'extraire les données recueillies en Lettres.

Tableau 2. Bilan du travail de compréhension orale

1 - Satisfaction en ce qui concerne 


\begin{tabular}{|c|c|c|}
\hline L'usage des 3 cassettes individuelles & $72 \%$ & $75 \%$ \\
\hline Le thème des documents oraux proposés & $64 \%$ & $68 \%$ \\
\hline Le nombre de documents (ou le volume) & $58 \%$ & $63 \%$ \\
\hline La difficulté (acceptable) de compréhension & $58 \%$ & $64 \%$ \\
\hline Les fiches de travail en tant qu'aide à la compréhension & $76 \%$ & $75 \%$ \\
\hline $\begin{array}{l}2 \text { - En combien de séances avez-vous travaillé chaque document } \\
\text { hebdomadaire? }\end{array}$ & & \\
\hline En 1 fois & $54 \%$ & \\
\hline 2 ou 3 séances & $40 \%$ & \\
\hline 4 séances & $4 \%$ & \\
\hline $\begin{array}{l}3 \text { - Combien de temps avez-vous consacré au travail de compréhension orale } \\
\text { de chaque document hebdomadaire? }\end{array}$ & & \\
\hline moins de $30 \mathrm{~min}$ & $1 \%$ & \\
\hline entre $30 \mathrm{~min}$ et $1 \mathrm{~h}$ & $16 \%$ & \\
\hline entre 1 et $2 \mathrm{~h}$ & $55 \%$ & \\
\hline entre 2 à $3 \mathrm{~h}$ & $23 \%$ & \\
\hline plus de $3 \mathrm{~h}$ & $3 \%$ & \\
\hline Durée moyenne en 93-94 & $1 \mathrm{~h} 43 \mathrm{~min}$ & \\
\hline Durée moyenne en 94-95 & $1 \mathrm{~h} 22 \mathrm{~min}$ & \\
\hline
\end{tabular}

En matière de stratégie d'écoute, les réponses apportées sur les critères d'objectifs à atteindre sont autant d'indices qui permettent de mieux connaître les stratégies utilisées, de les formaliser en discussion de méthodologie; d'autant que ces indices varient sensiblement selon le niveau initial de l'étudiant ou le niveau atteint en fin d'année.

Ce dernier aspect est le plus intéressant pour le didacticien-chercheur à la recherche d'une plus grande autonomie de l'apprenant, car l'individualisation du parcours d'apprentissage passe pour l'étudiant par une meilleure connaissance de soi-même en train d'apprendre, et par une adaptation de ce même parcours à des préférences ou dominantes de processus opératoires, définissant par ce fait une nouvelle fonction de l'enseignant-conseiller.

34 À la satisfaction des étudiants, s'ajoutait celle des 18 enseignants- intervenants des groupes concernés: un même contenu pour tous les groupes, la systématisation de la méthode, de la fréquence, l'aspect quantitatif du contenu, une impression de progrès dans la capacité à comprendre, et pour beaucoup la concrétisation d'une individualisation 
de la tâche d'écoute (en 1 an, 800 étudiants ont consacré en moyenne $41 \mathrm{~h}$ à une écoute individuelle guidée, et ce, en dehors de l'établissement).

Cependant, malgré le constat d'uniformité et d'« efficacité » en acquisition langagière, les caractéristiques de l'acte d'apprentissage sont restées les mêmes : l'institution décide de l'enseignement que va suivre l'étudiant, elle fixe les objectifs, elle détermine les moyens, les modalités d'exécution, elle détermine l'évaluation, principalement du contenu, et non du processus. Si elle élargit le lieu ou l'espace par un travail en autonomie à la maison, celui-ci n'est qu'une extension de l'activité présentielle.

L'autonomie s'arrête là, l'apprenant exécute des tâches demandées mais ne prend pas part à la décision ou à la construction de son programme ni à la gestion de son temps, ni au choix des échéances. Pour efficace que cette méthode ait été dans cette première phase d'expérimentation en réponse à l'institution, elle a cependant peu aidé l'étudiant à être autonome.

CRESSIDA, Centre de ressources, a répondu à une demande quantitative du savoir, en fournissant des matériaux ou connaissances de nature déclarative, dans des domaines thématiques successifs et atomisés, selon un mode de distribution systématique, molaire et indifférencié. C'est une étape utile et un des rôles des Centres de ressources. Cependant il ne suffit pas d'être exposé à la langue pour progresser; il ne suffit pas non plus d'individualiser la période d'écoute hors de l'université pour rendre le récepteur autonome.

\section{If a line was drawn from the Severn to the Wash}

La signification est le rapport entre un événement et un contexte, les lacunes ne sont pas uniquement des manques de connaissances linguistiques, mais aussi culturelles, par l'implicite inhérent à tout discours.

\section{1. Évolution du concept}

Le fondement de l'évolution du concept de Centre et de la nature des services proposés a été la formulation d'hypothèses de recherche fondées sur une approche cognitive de l'acquisition, et, ici, du perfectionnement de la compréhension.

Le cadre épistémologique général de référence est l'architecture cognitive d'Anderson qui prend en compte l'ensemble des fonctions mentales de l'homme: la perception, l'attention, la mémoire, le langage et les activités intellectuelles: raisonnement et résolution de problèmes, formalisation, et construction de concepts.

D. Gaonac'h (1990) écrit que la psychologie cognitive cherche aussi «à rendre compte de l'ensemble des mécanismes, conscients ou inconscients, contrôlés ou automatisés, à l'œuvre dans les activités mentales ». Cette approche est pluridisciplinaire : le traitement du langage n'est qu'un des aspects du fonctionnement général de l'esprit: conception partagée par des philosophes, des linguistes et des anthropologues, des spécialistes de l'intelligence artificielle, et surtout des neurosciences: la psychologie cognitive croit de plus en plus à l'équation identitaire : cognitif = neuronal.

42 L'activité cognitive vise à produire un ordre dans l'environnement de l'être : connaître revient à classer, ranger, orienter, construire des configurations stables; elle privilégie l'organisation et la structuration des situations, puis des savoirs manipulés, s'oppose au 
béhaviorisme skinnérien qui, lui, est dépendant de l'environnement. Les connaissances nouvelles sont bâties à partir des connaissances disponibles, par des mécanismes de catégorisation, de transfert analogique et d'induction: comprendre est un acte dynamique (Bransford \& McCarrell 1975).

Comprendre "C'est construire du sens, non des formes linguistiques» (Holec 1990). Le sens est le produit d'un système de traitement de l'information (théorie de Shannon et Weaver). Le contexte socio-culturel fournit les significations. L'individu, par la causalité, l'espace et le temps (influence de Kant), organise et structure les situations.

Du point de vue de la linguistique, comprendre, c'est saisir un ancrage dans une situation (cf. l'acte d'énonciation de Benveniste).

Le modèle de récepteur est un modèle principalement onomasiologique : comprendre c'est faire des hypothèses et les vérifier, c'est anticiper. Lindsay et Norman (1977) ajoutent que l'on ne peut anticiper que si on a de l'expérience, c'est-à-dire des connaissances préalables. Les psycholinguistes, dont J. Caron (1989), disent en substance : « je comprends ce que j'attends ».

Comprendre c'est établir une relation entre les percepts et les catégories abstraites que nous possédons: Le Dictionnaire Robert définit ainsi le premier sens de comprendre: «Embrasser dans un ensemble. Faire entrer dans un tout, une catégorie ».

\subsection{Conséquences méthodologiques : la réponse ACADEME}

Le didacticien doit permettre de faire progresser l'étudiant dans sa perception de l'information, dans son traitement, et sa capacité d'abstraction, d'où les 2 orientations méthodologiques suivantes :

Un document sonore est "facile» si le récepteur dispose de suffisamment de connaissances pour atteindre son objectif d'écoute. Nous pourrions paraphraser la phrase de David Marr (1982) à propos du percept visuel : « Percevoir, c'est savoir en regardant ce qui est où. », nous pensons que c'est au Centre de ressources de proposer des documents facilitant l'accès à la compréhension.

De cette exigence sont nés le concept et la production ACADEME (Apports et Convergences Au-Delà Des Médias), en cours de validation.

50 Le produit proposé est une feuille de format A4, hebdomadaire, complémentaire de chaque document audio étudié; (illustration en annexe). Cette feuille présente une combinaison :

51 - de définitions opératoires simples, puis élaborées, pour constituer une condition favorable d'entrée dans la représentation de l'émetteur du message à comprendre.

52 - d'apports factuels minimaux sur le thème qui fait l'objet d'apprentissage de compréhension : des graphiques ou des documents de presse écrite relatant soit la même information, soit une analyse ou un prolongement du thème ou de la réalité culturelle abordée et qui se veulent des facilitateurs d'anticipation.

53 La feuille ACADEME introduit ainsi la thématique, place l'événement dans son contexte géographique et culturel, rend un lexique minimal familier, active un réseau sémantique, et constitue un amorçage conceptuel de celui-ci, afin de permettre « une représentation intégrée et cohérente », expression de Bruner (1975). 
54 L'enquête auprès des étudiants révèle que ceux-ci ont consacré 51 minutes en moyenne à cette lecture préalable; que les avantages de cette lecture sont, par ordre décroissant d'importance: le contexte, le vocabulaire, les mots-clés, l'anticipation, l'intention des locuteurs, et l'identité des intervenants.

55 À titre d'illustration, voici quelques données sur l'influence de la lecture de 2 articles de presse sur «la popularité de la famille royale», préalable au travail de compréhension d'un document VIFAX sonore relatant la même information:

56 - Mots identifiés dans le document oral, parce qu'ils avaient été lus auparavant : sur 38 étudiants : moyenne : 20,3 mots

57 - Idées (ou propositions linguistiques) identifiées dans le document oral grâce à la lecture préalable : en moyenne 6,2 sur 17 énoncés attendus dans la grille de compréhension.

58 - Temps consacré à la lecture de ces textes : en moyenne : 52 minutes (de 12' à 120', selon les étudiants)

59 - Temps consacré à la tâche d'audition du document VIFAX : en moyenne : 77' (de 40' et 120 ', selon les étudiants)

60 Après 2 années d'expérimentation, nous sommes en mesure d'affirmer que l'apport conjugué de définitions, de mots-clés en situation, d'extraits de presse écrite sur le même thème, sont les supports nécessaires et suffisants d'une médiation vers l'intelligibilité d'un document oral, au niveau de DEUG.

\subsection{Vers une modification des activités présentielles}

61 Le recours systématique à des définitions multiples en provenance de sources variées initie à la catégorisation, à la méthode de classement des connaissances, et progressivement au rattachement de toute nouvelle acquisition à un schéma organisé conceptuel et abstrait. Débarrassé de tâches d'audition, opérations de bas niveau, l'institution peut ainsi se consacrer à la méthodologie : l'objectif des séances in situ va devenir qualitatif et procédural - apprendre à apprendre. Notre mise en œuvre est la suivante :

\section{Les 3 phases de mise en œuvre}

Première phase présentielle: anticipation seul et à 2, lecture de l'image (polysémie, connotation, dénotation, synecdoque), activité sociale, interactive; conflit cognitif (référence à Vygotski), activité pragmatique et langagière: de la compréhension à la production-vérification. Cette connaissance préalable qui permet à l'apprenant d'attendre tel champ lexical, telle proposition linguistique, et d'optimiser ses possibilités d'hypothèses, illustre le paradigme «d'amorçage conscient " d'Anderson: la mise en place d'une stratégie anticipatrice, une "focalisation d'attente » dit M.T. Vasseur, une propagation de l'activation une organisation à l'avance. C'est déjà une activité conceptuelle, un processus de haut niveau.

63 Une phase individuelle : une tâche de segmentation, de raisonnement, d'inférence ; une action finalisée ; une tâche individualisée d'appropriation du matériau, une tâche guidée : du plus simple au plus complexe, à l'aide de deux documents écrits d'accompagnement : D. Gaonac'h (1990) nous dit que l'effort d'attention est plus effort quand l'apprenant attend une information pertinente.

Une deuxième période collective, au début du cours suivant, avec les activités suivantes : 

d acquisition. À ce sujet les adultes sont demandeurs de guidage, y compris ceux qui on acquis une compétence élevée d'organisation : parmi les attentes d'une communauté de 128 chercheurs scientifiques (enquête à paraître), $33 \%$ considèrent comme prioritaire « l'analyse par un enseignant de L'UFR, de leurs besoins de langue, et la proposition d'une stratégie d'acquisition en autonomie guidée, à leur rythme ».

- avec un public LANSAD : poursuite de l'étude du thème, par résolutions de problème, ou jeux de rôle, tâches de plus longue haleine (telle que la confection collective d'un glossaire de langue de la spécialité), réflexion sur la langue : activités interlangues, etc.

En résumé, le présentiel prépare une période autonome, puis favorise l'organisation des connaissances relationnelles et conceptuelles, en vue de la construction scientifique d'un savoir (déclaratif et procédural).

\section{Le nouveau rôle du Centre de ressources}

72 Aux rôles précédemment cités de traitements matériel et intellectuel, nous ajoutons les missions didactiques suivantes.

Fournir une connaissance préalable par des définitions simples qui délimitent la thématique

73 C'est une tâche de contextualisation, au sens de connaissances référentielles extralinguistiques, de « terrain commun » (référence à Clark et Murphy 1982), de configuration d'activation d'un grand réseau sémantique à un moment donné, de déictique, de thématisation et focalisation au sens linguistique, une " dévolution du problème », dirait Brousseau (1986).

Ce Centre de ressources est, selon Hubert Fondin (1992), «un intermédiaire entre utilisateur et sources ». Il vulgarise, il apprête l'information, il la rend accessible, mais il ne cherche pas à simplifier une réalité complexe.

Ces deux aspects impliquent l'usage d'autres sources de documents : nous avons choisi 4 types de dictionnaires, le CD-Rom Grolier, et le quotidien The Independent. Une analyse systématique de six mois de parution de ce quotidien a abouti à un répertoire de 3400 articles sélectionnés et classés selon les mêmes critères que les documents VIFAX, qui permet pour la totalité des documents vidéo retenus de constituer spontanément des dossiers thématiques disponibles actuellement pour des groupes, pour des enseignants d'autres filières, et demain pour des étudiants en libre accès. À titre d'exemple, nous avons établi des liens entre les 14 documents VIFAX retenus pour le programme d'une année en DEUG scientifique B 2e année, et 221 articles de The Independent, de la même période de diffusion.

76 Il s'agit d'une tâche certes technique, mais surtout intellectuelle: un état permanent d'alerte ou de veille et de curiosité. Le linguiste responsable du Centre, par sa 
connaissance de la culture anglo-saxonne, déchiffre le signe ou le repère culturel: il déchiffre, à la façon des herméneutes qui voulaient réduire l'écart entre le monde de l'auteur du message et celui de l'auditeur, il nomme le concept qu'il perçoit, sous forme de mots-clés ne figurant pas dans le document, il redevient Callimaque.

Au-delà des tâches de "courtier en information » selon l'expression d'Hubert Fondin (1992), les liens nouveaux, les mises en relation de documents multisources, sont chaque semaine des élaborations de ce que Ch. Puren (1994) appelle des « cohérences partielles, temporaires et plurielles »: c'est donc une création originale d'ingénierie didactique, une nouvelle technologie intellectuelle.

La feuille A4 n'est pas et ne sera jamais un document de professionnel de la PAO : elle est imparfaite et inachevée : au-delà de l'apport systématique lexical minimal, elle doit être déclencheur de curiosité, ouvrir des chapitres différents, évoquer des analogies et des proximités, des paquets d'information fragmentée, elle est multifenêtre; elle a la cohérence temporaire (ou l'incohérence) d'une démarche hypertextuelle; d'ailleurs pour combien de temps encore cette production sera-t-elle sur papier? L'aboutissement de ce tissage de relations est bien sûr l'intégration multisources en un logiciel d'auteur.

\section{Conclusion}

En somme, au fur et à mesure que les tâches de compréhension et de lecture extensive de préparation à l'audition individuelle sont possibles à l'extérieur de l'institution (et il est souhaitable que ces activités aient lieu sans notre présence, si elles sont guidées), le temps ainsi libéré permet d'orienter la majeure partie des activités présentielles vers des pratiques procédurales qui préparent à l'autonomie le futur chercheur, le futur professeur des écoles, le futur ingénieur, mais aussi le futur citoyen. Ces pratiques aident à développer la capacité de s'orienter, de se repérer dans la masse informationnelle de notre époque, et à participer à l'organisation du monde. "Une organisation telle qu'il puisse le comprendre, agir sur lui, s'y adapter ou s'en évader » dit Roqueplo (1974).

80 Les nouvelles technologies éducatives nous inondent de savoirs atomisés. Comment l'adulte issu du système universitaire se prépare-t-il à cette "crue » informationnelle qu'est l'accès immédiat et surabondant des données de CD-Rom et des réseaux d'information? Qu'advient-il de la linéarité de nos acquisitions et de notre culture face à l'hypernavigation et à l'aléatoire décontextualisé du CD-Rom? Que deviennent enfin les repères anthropologiques fondamentaux que sont l'appartenance à un « Territoire », et la notion du temps?

81 «L'Espace Marchand», selon le terme de Pierre Lévy (1994), dans lequel nous devons ranger les NTE, rend encore plus vitale la réflexion sur le concept de « topologie » dont la définition du Robert est la suivante : « ... étude des propriétés géométriques se conservant par déformation continue puis généralisée pour englober les notions de limite et de voisinage ». Si nous cherchions une métaphore de circularité nous dirions que l'acte de définition est l'alpha et l'omega de la démarche méthodologique en langues, en milieu d'adultes : en effet, indispensable à la thématisation et à la focalisation dans l'étape de compréhension, il est aussi l'aboutissement d'une formulation collective de nouvelles connaissances partagées : il oblige à remonter au générique, au prototype, au concept primaire d'appartenance, aux liaisons inter-concepts, il est la mise en texte d'une 
configuration temporairement stable ; d'ailleurs dans l'étymologie du mot « texte », il y a le mot tissu, le tissage de liens... nous retrouvons « Internet ».

Notre opération d'assemblage d'outils méthodologiques se veut une "nouvelle technologie intellectuelle» tendant vers l'autonomie. Dans tous les colloques sur l'autonomie, on rappelle la troisième définition du mot « autonomie » dans le Dictionnaire Robert : «Distance que peut franchir un véhicule un avion, un navire sans être ravitaillé en carburant ». Le Centre de ressources, médiation entre les sources et les utilisateurs, peut contribuer à une reconstruction permanente de la Carte du Tendre.

Au fond, la question qui se pose au didacticien pourrait être : à quelle condition l'homme sédentaire peut-il redevenir « nomade » ?

\section{BIBLIOGRAPHIE}

Bransford, J. D. et N. S. McCarrell. 1975. « A sketch of a cognitive approach to comprehensio ». In Cognition and the Symbolic Processes. New York :Wiley.

Caron, J. 1989. Précis de psycholinguistique. Paris : Presses Universitaires de France.

Fondin, H.1992. Rechercher et traiter l'information. Paris : Hachette.

Gaonac'h, D. 1990. « Table Ronde ». Le Français dans le Monde, « Acquisition et utilisation d'une langue étrangère ", numéro spécial, février-mars.

Holec, H. 1990. « La compréhension orale : un processus et un comportement ». Le Français dans le Monde, « Acquisition et utilisation d'une langue étrangère », numéro spécial, février-mars.

Levy, P. 1994. L'intelligence collective. Pour une anthropologie du cyberspace. Paris : Éditions La Découverte.

Marr, D.1982. Vision. San Francisco : Freeman.

Puren, C. 1994. «Éthique et didactique scolaire des langues ». Langues Modernes 2, 55-62.

Roqueplo, P. 1974. Le partage du savoir. Paris : Éditions du Seuil.

\section{ANNEXES}

\section{Annexe : Dossiers pédagogiques ACADEME}

NDLR : N’est reproduit ici que le dossier L13 


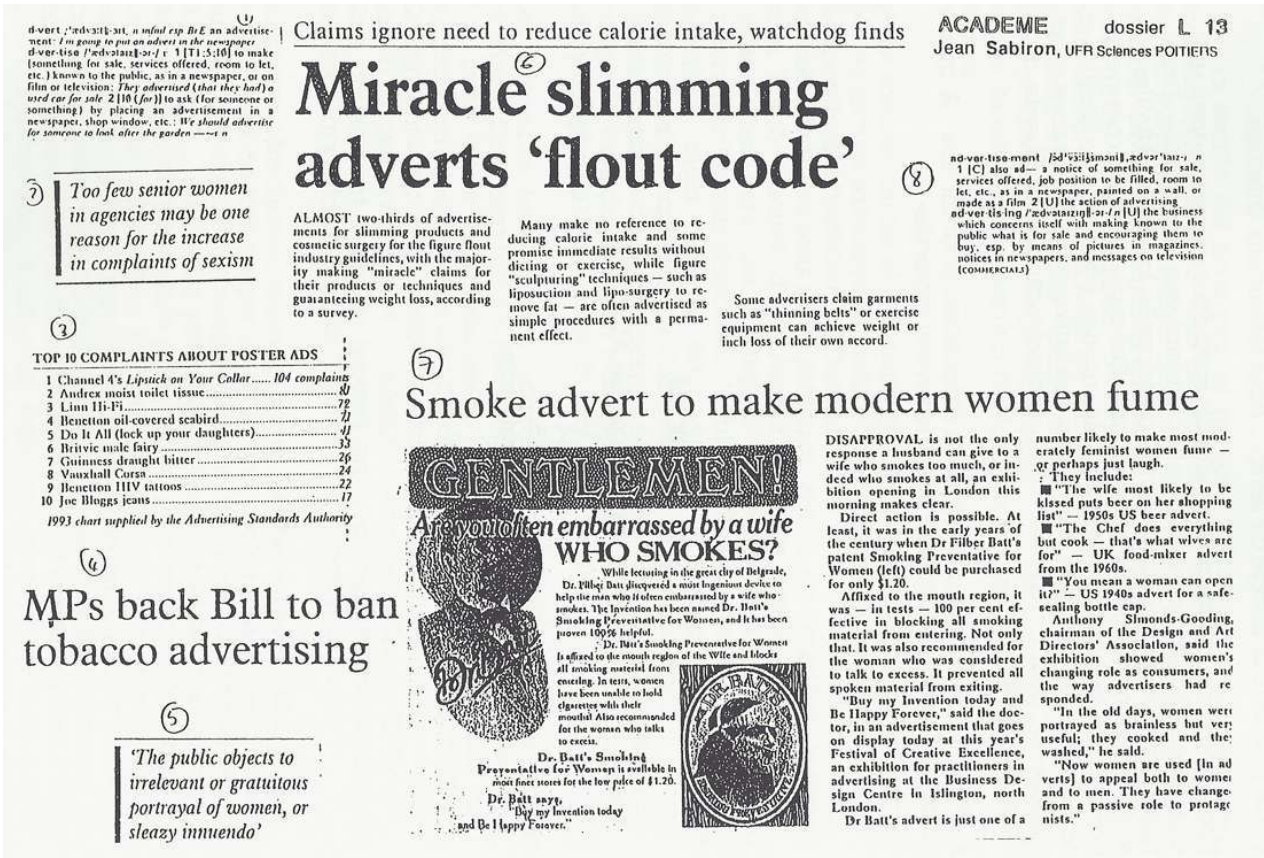

\section{NOTES}

1. Depuis tout récemment, sous forme de « document annexe » à un courrier électronique.

\section{RÉSUMÉS}

Nous relatons dans cet article l'évolution de la nature des prestations d'un Centre de ressources : d'abord, réponse à une demande langagière par la fourniture de documents audiovisuels en vue d'une exposition raisonnée à la langue anglaise authentique, il devient la proposition d'une approche méthodologique multisources intégrée. Celle-ci, de caractère pragmatique, vise à une contextualisation linguistique et extra-linguistique des documents oraux faisant l'objet d'étude, et à la structuration des nouveaux acquis dans une perspective cognitive.

This study considers the changing role of a Resource Centre at university level: this first tried to provide teachers with authentic oral material for comprehension; then it offered both teachers and students guidance by the gathering of linguistic and extra-linguistic contextual elements. Finally, it developed into a combination of pragmatic tools and a cognitive methodological preparation of students, in order to achieve a more efficient organisation of knowledge. 
INDEX

Mots-clés : ACADEME, aide à l'apprentissage, compréhension, contextualisation, intégration, matériel

Keywords : contextualization, facilitating, material

\section{AUTEUR}

\section{JEAN SABIRON}

Membre du GERAS et de RANACLES, Jean Sabiron enseigne à l'UFR Sciences de Poitiers. Son domaine de recherche concerne l'intégration des supports audiovisuels et de la presse écrite dans une perspective de didactique s'adressant à des grands groupes (DEUG-Langues ou DEUGLANSAD). Il a soutenu à Bordeaux 2 une thèse sur ce sujet en décembre 1995.

jean.sabiron@orange.fr 